\title{
Pathologische Veränderungen der Muskeln bei Cholera
} asiatica.

Von Dr. W. Warasi, 1. Assistent am Pathologisch-anatomischen Institut der Reichs-Universität in Tiflis (Georgia).

Unter dem Einflusse des Choleratoxins treten in fast allen Organen pathologische Vevänderungen auf. Nur bezüglich der Muskulatur behauptet $\mathrm{Kr}$ raus, daß die asiatische Cholera hier weder makroskopische, noch mikroskopische Veränderungen schafft.

$\mathrm{DaB}$ dem nicht so ist, haben mich meine Untersuchungen gelehrt; ich fand bei frischen Choleraleichen in den Muskeln trübe Schwellung und wachsartige Degeneration. Letztere ist zuerst von Zenker im $M$. rectus abdominis und im $M$. adductor femoris bei Abdominaltyphus entdeckt worden; später fand man sie auch bei anderen Infektionskrankheiten (Scharlach, Flecktyphus, Diphtherie) und bei Trichinose, und es ist zweifellos, daB diese Muskelveränderungen auf Toxinwirkungen beruhen.

Mein erster Fall betraf die Leiche emes etwa 25jährigen Mannes, der 3-4 Stunden nach seiner Erkrankung an Cholera starb. Sektion 2 Stunden später. Es wurden $M$. rectus abdominis und $M$. gastrocnemius untersucht. In letzterem fand ich deutliche wachsartige Degeneration der Muskelfasern und die gleiche Veränderung - nur in geringerem Grade - im $M$. rectus abdominis.

Im zweiten Falle handelte es sich um einen 55jährigen, am 15. Tagc der Erkrankung im Stadium typhosum zugrundegegangenen Mann. Hier war in den obengenannten Muskeln trübe Schwellung festzustellen.

$\mathrm{Ob}$ konstant solche Veränderungen bei Cholera sich finden, müssen weitere Untersuchungen zeigen. Vorläufig kann ich" nur" sagen," daß in manchen Fällen von Cholera asiatica die quergestreiften Muskeln pathologische Veränderungen erleiden in Form der trüben Schwellung und der wachsartigen Degeneration. 